\title{
CRISPR/Cas9-mediated cervical cancer treatment targeting human papillomavirus E6
}

\author{
TAKAHIRO YOSHIBA ${ }^{1,2}$, YASUSHI SAGA ${ }^{1,2}$, MASASHI URABE $^{1}$, RYOSUKE UCHIBORI $^{1}$, \\ SHIGEKI MATSUBARA ${ }^{2}$, HIROYUKI FUJIWARA ${ }^{2}$ and HIROAKI MIZUKAMI $^{1}$ \\ ${ }^{1}$ Division of Genetic Therapeutics, Center for Molecular Medicine; ${ }^{2}$ Department of Obstetrics and Gynecology, \\ Jichi Medical University, Shimotsuke, Tochigi 329-0498, Japan
}

Received June 21, 2018; Accepted November 30, 2018

DOI: $10.3892 / \mathrm{ol} .2018 .9815$

\begin{abstract}
High-risk human papillomavirus (HPV) is a common cause of cervical cancer. HPV E6 oncoprotein promotes the degradation of host tumor suppressor gene $p 53$, leading to the development of tumors. Therapeutic strategies that specifically target E6, which is constitutively expressed in tumors and is not present in normal tissues, may be highly effective and safe. CRISPR-CRISPR associated protein 9 (Cas9) is one of the genome editing technologies that has recently garnered attention, and is used to knockout target gene expression. By combining cervical cancer cell lines engineered to constitutively express Cas9 and an adeno-associated virus (AAV) vector carrying a single guide (sg) RNA targeting E6 (AAV-sgE6), the present study sought to investigate the effects of this novel therapeutic approach on cervical cancer. The Cas9 gene was transfected into three high-risk HPV-positive cervical cancer cell lines (HeLa, HCS-2, and SKG-I) to establish cell lines that constitutively expressed Cas9. Using these cell lines, genetic mutations and their frequencies, as well as the levels of protein expression, apoptosis and cell proliferation were examined in vitro. In addition, the effects of AAV-sgE6 were examined in a mouse model of cervical cancer in vivo by a single administration of AAV-sgE6 directly into subcutaneous tumors. The results demonstrated that multiple mutations occurred frequently in the targeted E6 genomic sequence in cervical cancer cells transduced with AAV-sgE6. In addition, these AAV-sgE6-transduced cells had reduced expression of E6, increased expression of p53, increased apoptosis and their growth was suppressed in a concentration-dependent manner.
\end{abstract}

Correspondence to: Dr Yasushi Saga or Dr Hiroaki Mizukami, Division of Genetic Therapeutics, Center for Molecular Medicine, Jichi Medical University, 3311-1 Yakushiji, Shimotsuke, Tochigi 329-0498, Japan

E-mail: saga@jichi.ac.jp

E-mail:miz@jichi.ac.jp

Key words: adeno-associated virus vector, cervical cancer, clustered regularly interspaced short palindromic repeats-CRISPR associated proteins 9, E6, high-risk human papilloma virus
Furthermore, subcutaneous tumor growth was significantly suppressed in vivo following intratumoral administration of AAV-sgE6, and adverse events due to AAV-sgE6 administration were not observed. Collectively, the present results indicated that targeting E6 expression in high-risk HPV by CRISPR-Cas9 is a highly specific and effective strategy that may be effective in treating patients with cervical cancer.

\section{Introduction}

Cervical cancer is the second most common cause of cancer-related deaths in women worldwide (1). Each year, approximately 500,000 women are diagnosed with cervical cancer, and 270,000 will die from it (1). Patients with advanced cervical cancer are treated with surgery, radiation therapy, chemotherapy, or a combination of these strategies. However, the prognosis of advanced cervical cancer remains poor, with no significant improvements in the overall treatment outcome over the last three decades (2). Cervical cancer is caused by high-risk human papillomavirus (HPV) infection (3-5). Specifically, persistent infection of the basal epithelial cells of the cervix by high-risk HPV causes the integration of the HPV genome into the host chromosome, which leads to the expression of the HPV-E6 oncoprotein that inactivates the tumor suppressor gene $p 53$, resulting in tumor formation $(6,7)$. Thus, suppressing the expression of E6 may lead to the treatment of cervical cancer. Furthermore, the expression of E6 is limited to cervical lesions, with no expression in healthy tissue, including the cervix (8). Therefore, specific targeting of E6 expression is likely a safe strategy that would spare normal tissue.

CRISPR-Cas9 is one of the genome editing technologies that has gained much attention in recent years. It induces double stranded breaks (DSBs) at specific target DNA locations by the combined action of a single guide RNA (sgRNA) that recognizes a specific DNA sequence and the Cas9 nuclease that induces DSBs (9). During the repair of these DSBs by non-homologous end joining, gene mutations often occur in the form of base insertions or deletions. Expression of a gene can be knocked out if these mutations occur in the coding region of the gene (10). Thus, CRISPR-Cas9 can be used to induce the specific knockout of E6 expression in cervical cancer cells. However, application of such a strategy in clinical practice requires an effective vector that enables gene transfer into targeted cervical cancer cells. 
Adeno-associated virus (AAV) vector is an attractive option for gene transfer as it is derived from a non-pathogenic virus and can induce gene transfer in non-dividing cells (11). Previous studies have demonstrated prolonged transgene expression and clinical benefits following the direct administration of the AAV vector into humans (12). Several serotypes of AAV vectors with varying gene transfer efficacy for specific tissues and organs have been reported. For example, AAV serotypes 1 and 7,2 and 3,5, and 8 are effective in gene transfer in the skeletal muscles, nerves, and liver, respectively (13). We previously reported that the AAV type 2 vector was the most effective in inducing gene transfer into cervical cancer cells (14).

There are no treatment strategies for cervical cancer that target high-risk HPV E6. In the present study, we performed in vitro and in vivo experiments to develop a CRISPR-Cas9-based, effective, and highly specific therapy targeting high-risk HPV E6 for cervical cancer.

\section{Materials and methods}

Cell culture. HPV 18-positive human cervical cancer cell lines (HeLa, HCS-2, SKG-I), and human immortal cell line 293 were purchased from the Japanese Collection of Research Bioresources Cell Bank (JCRB, Osaka, Japan). These cells were cultured in Dulbecco's Modified Eagle Medium/F12 (DMEM/F12; Thermo Fisher Scientific, Inc., Waltham, MA, USA) supplemented with $10 \%$ inactivated fetal bovine serum (FBS; Sigma-Aldrich; Merck KGaA, Darmstadt, Germany) and $1 \%$ penicillin/streptomycin (Thermo Fisher Scientific, Inc.) at $37^{\circ} \mathrm{C}$ under $5 \% \mathrm{CO}_{2}$.

Construction of the plasmid vector. The part of the CMV promotor and Cas9 which was tagged with human influenza hemagglutinin (HA) were cut out from the SpeI and XbaI site of pRGEN-Cas9-CMV (Toolgen, Seoul, South Korea) and inserted into the SpeI and XbaI sites of pCMV-IRES-bsr (15) to produce the Cas9-expression vector (pCMV-Cas9-HA-IRES-bsr).

Optimized CRISPR Design (crispr.mit.edu/) was used to search for the HPV18 E6 sequence to be targeted by CRISPR. The sequence with the highest score was selected. Two DNA oligonucleotides, 5'-CACCGGAGCTTGTAG GGTCGCCGTGT-3' and 5'-AAACACACGGCGACCCTA CAAGCTC-3', were annealed and inserted at the BsaI site of pRGEN-U6-sgRNA (Toolgen) to produce a vector expressing an sgRNA targeting E6 (sgE6) (pRGEN-U6-sgE6). Two inverted terminal repeats (ITRs) cleaved from pWlacZ (16) were then added to the vector to produce pW-U6-sgE6. Two ITRs cleaved from pWlacZ were also added to pRGEN-U6-sgRNA to produce pW-U6-sgRNA, which was used as a control vector.

Establishment of Cas9-expressing cervical cancer cell lines. HeLa, HCS-2, and SKG-I cell lines were transfected with pCMV-Cas9-HA-IRES-bsr using Lipofectamine LTX and Plus Reagent (Invitrogen; Thermo Fisher Scientific, Inc.). Transfected cells were selected by culturing in cell culture media containing $10 \mu \mathrm{g} / \mathrm{ml}$ of Blasticidin S Hydrochloride (Funakoshi, Tokyo, Japan) to collect single colonies.
Cell growth curve. Tumor cells were plated onto 96-well plates (500 cells/well), and $10 \mu 1$ of Premix WST-1 Cell Proliferation Assay System (Takara Bio Inc., Tokyo, Japan) was added to each well every $24 \mathrm{~h}$. Absorption was measured at $450 \mathrm{~nm} 24 \mathrm{~h}$ after premix added using SpectraMax 190 (Molecular Devices, LLC, Sunnyvale, CA, USA) to produce a cell growth curve.

$A A V$ vector preparation. sgE6-containing and control AAV vectors were prepared by transfecting the 293 cells with 3 plasmids, including pW-U6-sgE6, or pW-U6-sgRNA, adenovirus helper plasmid (16), and AAV type 2 helper plasmid $(17,18)$ via calcium phosphate transfection, and cells were collected $72 \mathrm{~h}$ later. Cells were then exposed to three freeze-thaw cycles to obtain the recombinant AAV vectors. The solution containing the vectors was purified by cesium chloride density gradient centrifugation, and the vector titer was measured by reverse transcription-quantitative polymerase chain reaction (RT-qPCR) as described previously (19).

Detection of mutations in the E6 genome. Cas9-expressing cells were seeded onto 6-well plates at a concentration of $5 \times 10^{4} \mathrm{cells} /$ well, and were transduced $24 \mathrm{~h}$ later with AAV-sgE6 $\left(1 \times 10^{5}\right.$ viral genomes $\left.(\mathrm{vg}) / \mathrm{cell}\right)$. Cells were collected by trypsin $48 \mathrm{~h}$ later, and DNA was extracted according to the protocol using the QIAamp ${ }^{\circledR}$ DNA Mini kit (Qiagen GmbH, Hilden, Germany). The extracted DNA was used as a template to perform PCR using TaKaRa Ex Taq Hot Start version (Takara Bio Inc.) and PTC-100 (Bio-Rad Laboratories, Inc., Hercules, CA, USA) to amplify the E6 genome. The following primers were used for the reaction: Forward: 5'-GGGAGTGACCGA AAACGGTC-3', reverse: 5'-GTGTTTCTCTGCGTGTTG T-3'. PCR was carried out using 40 cycles of heating at $95^{\circ} \mathrm{C}$ for $30 \mathrm{sec}, 56^{\circ} \mathrm{C}$ for $30 \mathrm{sec}$, and $75^{\circ} \mathrm{C}$ for $30 \mathrm{sec}$. The PCR product was cloned according to the protocol using the Mighty TA-cloning kit (Takara Bio Inc.), and Sanger sequencing was performed using the Applied Biosystems 3730xl DNA Analyzer (Thermo Fisher Scientific, Inc.).

T7 Endonuclease 1 (T7E1) assay. Tumor cells were seeded onto 6-well plates at $5 \times 10^{4}$ cells/well, incubated for $24 \mathrm{~h}$, and transduced with either AAV-sgE6 or AAV-sgNC at $1 \times 10^{5} \mathrm{vg} / \mathrm{cell}$. Cells were collected by trypsin $48 \mathrm{~h}$ later, and DNA was extracted according to the protocol using the QIAamp ${ }^{\circledast}$ DNA Mini kit. The extracted DNA was used as a template to amplify the E6 genome. Using the thermal cycler PTC-100, PCR products were denatured for $2 \mathrm{~min}$ at $95^{\circ} \mathrm{C}$, then cooled to $30^{\circ} \mathrm{C}$ over $10 \mathrm{~min}$, and annealed. Double-strand DNA was reacted with T7 Endonuclease (M0302S; New England BioLabs, Inc., Ipswich, MA, USA) at $37^{\circ} \mathrm{C}$ for $20 \mathrm{~min}$. Electrophoresis was performed with a $0.8 \%$ agarose gel, and imaged using the BioDoc-It ${ }^{\circledR}$ Imaging System (UVP, Inc., Upland, CA, USA).

$R T$-qPCR. Tumor cells were seeded onto 6-well plates at $5 \times 10^{4}$ cells/well, incubated for $24 \mathrm{~h}$, and transduced with either $\mathrm{AAV}-\mathrm{sgE} 6$ or AAV-sgNC at $1 \times 10^{5} \mathrm{vg} / \mathrm{cell}$. Cells were collected by trypsin $48 \mathrm{~h}$ later, and DNA was extracted according to the protocol for the QIAamp ${ }^{\circledR}$ DNA mini kit. qPCR was performed according to the protocol using the Thermal Cycler Dice Real Time System II (Takara Bio Inc.). The PCR was carried out 
using 40 cycles of heating at $95^{\circ} \mathrm{C}$ for $15 \mathrm{~s}, 58^{\circ} \mathrm{C}$ for $15 \mathrm{~s}$, and $72^{\circ} \mathrm{C}$ for $20 \mathrm{~s}$.

Mutations in the E6 genome were measured by qPCR using a protocol adopted from a previous report (20). Primers with the following sequences were used: Mut primer forward: 5'-TTTGAGGATCCAACACGGCGA-3'. Mut primer reverse: 5'-GTCTTGCAGTGAAGTGCTCAG-3'. CTL primer forward: 5'-GTGCCRGAAACCGTTGAATCC-3'. CTL primer reverse: 5'-CCAGCTATGTTGTGAAATCGTCG-3'. To assess the validity of this assay, plasmid vectors containing non-mutated and mutated E6, as identified by Sanger sequencing, were used as templates to perform qPCR using two pairs of primers. Furthermore, the plasmid vectors mixed at varying ratios (10:0, 9:1, 8:2, 5:5, 2:8, 1:9, 0:10) were used as templates for qPCR to evaluate the quantitative ability of the assay. qPCR results were analyzed using the relative quantification (RQ) value (21), and the mutation rate was calculated as (1-RQ) x100 (\%).

Western blot analysis. Tumor cells were seeded onto 6-well plates at $5 \times 10^{4}$ cells/well, incubated for $24 \mathrm{~h}$, and transduced with either AAV-sgE6 or AAV-sgNC at $1 \times 10^{5} \mathrm{vg} / \mathrm{cell}$. Cells were lysed $48 \mathrm{~h}$ later using lysis buffer (1\% NP-40, $150 \mathrm{mM}$ $\mathrm{NaCl}, 50 \mathrm{mM}$ Tris-HCl, $\mathrm{pH} 8.0$ ), and extracted proteins were mixed with $1 \%$ sodium dodecyl sulfate (SDS) sample buffer (10 mM Tris-HCl, pH 7.5, 150 mM NaCl, 1\% SDS, EDTA-free Protease inhibitor cocktail (Roche, Basel, Switzerland), separated by electrophoresis using $5 \%$ (for $\mathrm{HA}, \mathrm{Rb}$ ) or $12.5 \%$ (for E6, p53, actin) polyacrylamide gels, and transferred onto polyvinylidene fluoride (PVDF) membranes (Merck KGaA, Darmstadt, Germany). Membranes were left at room temperature for $1 \mathrm{~h}$ in PVDF Blocking Reagent for Can Get Signal ${ }^{\circledR}$ (Toyobo Life Science, Osaka, Japan), washed three times using Tris-buffered saline-Tween-20 (TBS-T), and incubated overnight with the following antibodies at room temperature in Can Get Signal ${ }^{\circledR}$ Immunoreaction Enhance Solution 1 (Toyobo Life Science): Anti-HA-probe antibody (cat. no. sc-7392; Santa Cruz Biotechnology, Inc., Dallas, TX, USA), anti-HPV18E6 monoclonal antibody (cat. no. sc-365089; Santa Cruz Biotechnology, Inc.), anti-p53 monoclonal antibody (cat. no. sc-126; Santa Cruz Biotechnology, Inc.), anti-Rb monoclonal antibody (cat. no. 9313; Cell Signaling Technology, Inc., Danvers, MA, USA), and anti-actin polyclonal antibody (cat. no. A2066; Sigma-Aldrich; Merck KGaA). After the reaction, membranes were washed three times with TBS-T, and incubated with peroxidase-labeled anti-mouse or anti-rabbit antibodies (GE Healthcare Japan, Tokyo, Japan) in Can Get Signal ${ }^{\circledR}$ Immunoreaction Enhance Solution 2 (Toyobo Life Science) at room temperature for $1 \mathrm{~h}$. Membranes were then washed three times with TBS-T, incubated with ECL prime western blotting detection reagent (GE Healthcare Japan), and imaged using a cooled CCD system (LAS-4000mini: GE Healthcare Japan).

Apoptosis. Cells were seeded onto 12 -well plates at $5 \times 10^{4}$ cells/well, incubated for $24 \mathrm{~h}$, and transduced with either AAV-sgE6 or AAV-sgNC at $1 \times 10^{5} \mathrm{vg} / \mathrm{cell}$. The Apoptotic/Necrotic cells detection kit (PromoKine, Heidelberg, Germany) was used as per its protocol $24 \mathrm{~h}$ after the transduction, and FITC-Annexin V-positive cells were imaged using an inverted microscope (IX731; Olympus Corporation, Tokyo,
Japan). The number of FITC-positive cells was counted per high power field (HPF).

In vitro cell growth. Cells were seeded onto 96-well plates at 50 cells/well, and were transduced with either AAV-sgE6 or AAV-sgNC at $0-1 \times 10^{7} \mathrm{vg} /$ cell. Premix WST-1 cell proliferation assay system (Takara Bio Inc.) was added at $10 \mu \mathrm{l} /$ well $96 \mathrm{~h}$ after the transduction, and the absorbance was measured at $450 \mathrm{~nm} 24 \mathrm{~h}$ later using SpectraMax 190 (Molecular Devices, LLC).

In vivo experiments. Six to seven-week-old (15-20 g) Balb/c nude mice (CLEA Japan, Inc., Tokyo, Japan) were used for the in vivo experiments $(\mathrm{n}=8)$. SKG-I was selected among the three cervical cancer cell lines as it has been reported to induce tumors in nude mice. A total of $5 \times 10^{6}$ Cas9-expressing SKG-I cells (SKG-I/Cas9) were injected under the dorsal skin of nude mice. Simultaneously, $2 \times 10^{12} \mathrm{vg}$ AAV-sgE6 or AAV-sgNC were injected once in the same location. Tumor size was measured with calipers twice a week, and the tumor volume was calculated as length $\mathrm{x}$ width ${ }^{2} \times 1 / 2$. All mice were sacrificed by cervical dislocation when the diameter of tumors in the AAV-sgNC group reached over $20 \mathrm{~mm}$. Changes in body weight were measured, and gross observation of the injection site was performed at the experimental endpoint. DNA was extracted from tumors in both groups using the QIAamp DNA mini kit (Qiagen $\mathrm{GmbH}$ ), and the E6 mutation rate was measured by qPCR as described above. Multiple tumors were not observed in the present study.

Mice were grown under specific pathogen-free conditions. The experimental protocol was approved by the Jichi Medical University Ethics Committee (Tochigi, Japan), and strictly followed the National and Institutional Guidelines for animal experiments.

Statistical analysis. Statistical analysis was performed using SPSS v22 (IBM Corp., Armonk, NY, USA). Student's t-test was used to compare two groups. One-way analysis of variance with Bonferroni post-hoc test was performed to compare multiple groups. $\mathrm{P}<0.05$ was considered to indicate a statistically significant difference.

\section{Results}

Development of Cas9-expressing cervical cancer cell lines. In order to confirm that the Cas9 is introduced, we performed western blots for transfected cells using an anti-HA antibody. Cas9 protein was expressed only in those cells that received the Cas9 gene (Fig. 1A), confirming that Cas9-expressing cervical cancer cell lines HeLa/Cas9, HCS-2/Cas9, and SKG-I/Cas9 were established.

Cell growth curve. In order to assess the effect of Cas9 on cell growth we compared the growth of wild type and Cas9-expressing cervical cancer cell lines in vitro. As shown in Fig. 1B-D, there was no significant difference in cell growth between wild type and Cas9-expressing cells, indicating that Cas9 expression had no impact on cell growth in vitro.

Detection of E6 gene mutations. The E6 gene was sequenced by Sanger sequencing for HeLa/Cas9 cells $48 \mathrm{~h}$ after 

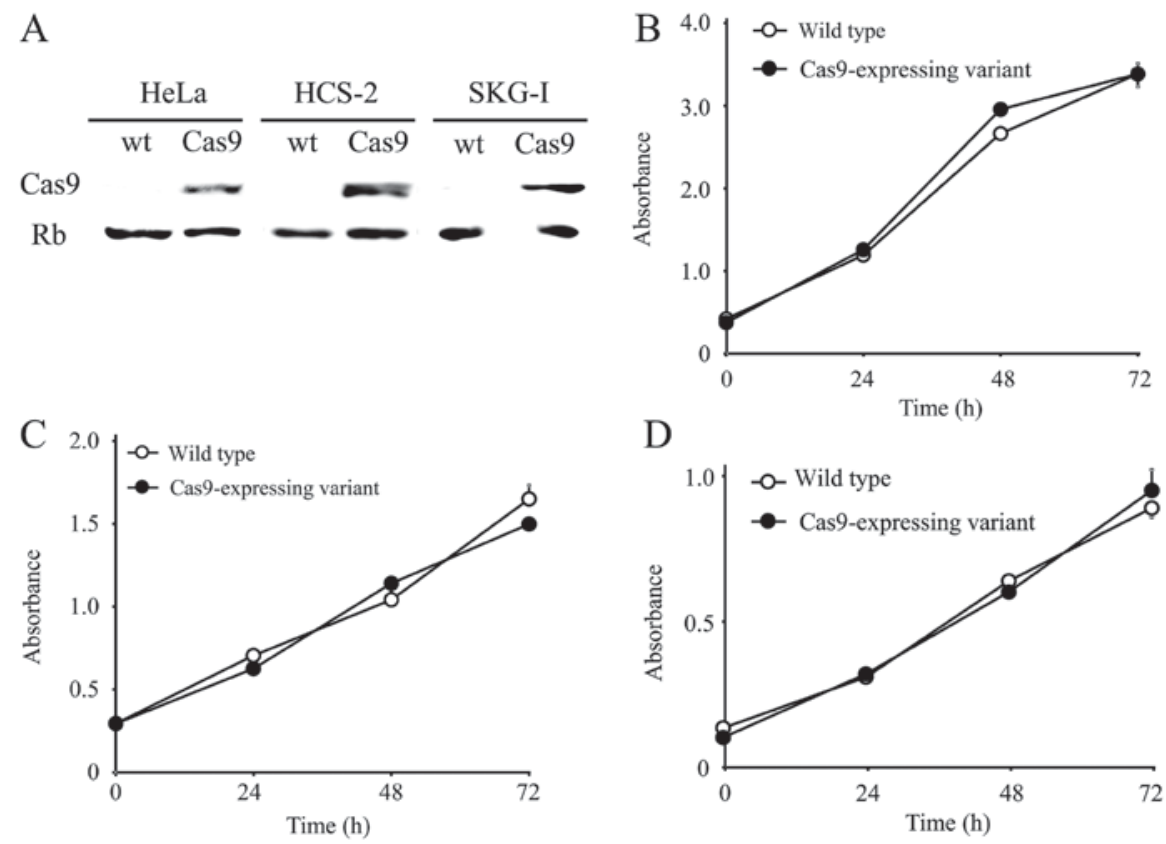

Figure 1. Development of Cas9-expressing cervical cancer cell lines. (A) Detection of Cas9 expression using western blotting. Cas9 was expressed only in the cell lines that received the Cas9 gene. Cell growth curve for Cas9-expressing (B) HeLa, (C) HCS-2 and (D) SKG-I cell lines. For all cell lines, there were no differences in the growth curve when compared with the respective wt cell lines. Results are expressed as the mean \pm standard deviation. Cas9, CRISPR associated protein 9; wt, wild-type; Rb, retinoblastoma.

AAV-sgE6 transduction. Several base insertions and deletions were identified mostly around the targeted region (Fig. 2A), indicating that genome editing was achieved for E6 in vitro by CRISPR-Cas9.

T7E1 assay. Products cleaved by T7E1 were detected only in cells transduced with AAV-sgE6 (Fig. 2B-D). Thus, gene mutations were introduced into the E6 gene by E6-targeting CRISPR-Cas9.

Measurement of mutation rates by $q P C R$. qPCR was performed using mutated plasmid vectors as templates, and demonstrated a significant increase in mutation rates (Fig. 2E). In addition, mutation rates increased with an increasing proportion of mutated plasmid vector, and this rate was equivalent to the proportion of the mutated vectors in the plasmid mixture (Fig. 2F).

The rate of E6 mutation was measured using the same method.Mutation rates were estimated to be $82 \%$ for HeLa/Cas9, $77 \%$ for $\mathrm{HCS}-2 / \mathrm{Cas} 9$, and $87 \%$ for SKG-I/Cas9 (Fig. 2G-I). Mutations were not found in untransduced cells or cells transduced with AAV-sgNC (Fig. 2G-I).

Western blot. E6 expression was detected in untransduced cells or cells transduced with AAV-sgNC. These cells did not express $p 53$. On the other hand, cells transduced with AAV-sgE6 had significantly decreased expression of E6, and significantly increased expression of p53 (Fig. 3A). These results indicated that E6 was knocked out effectively by CRISPR-Cas 9 targeting of E6, consequently increasing the expression of p53 at the protein level.

Apoptosis. Cells transduced with AAV-sgE6 had a significantly highernumberofFITC-Annexin V-positivecells(46.0 $\pm 5.0 / \mathrm{HPF})$ than untransduced cells $(1.0 \pm 1.4 / \mathrm{HPF})$ and cells transduced with AAV-sgNC $(0.8 \pm 1.3 / \mathrm{HPF})$ (Fig. $3 \mathrm{~B}$ and C). Thus, E6-targeting CRISPR-Cas9 induced apoptosis in tumor cells in vitro.

In vitro cell growth. For all cell lines, the number of live cells decreased with an increase in AAV-sgE6 vector dose (Fig. 3D). Thus, E6-targeting CRISPR-Cas9 suppressed tumor cell growth in a dose-dependent manner in vitro.

In vivo experiments. Tumor growth was suppressed significantly in mice injected with AAV-sgE6 as compared with those injected with AAV-sgNC (Fig. 4A). At the experimental endpoint of 42 days after the injection, tumors in the AAV-sgE6 group were significantly smaller than those in the AAV-sgNC group, at $114 \pm 60$ and $817 \pm 114 \mathrm{~mm}^{3}$, respectively (n=4/group, $\mathrm{P}<0.05$; Fig. 4B and C). Thus, E6-targeting CRISPR-Cas9 suppressed tumor growth in vivo. In addition, mutations in the E6 gene were not identified in the remaining tumors (data not shown). There was no significant difference in the body weights of mice measured prior to sacrifice (Fig. 4D), there were no abnormal findings in the subcutaneous tumor area (Fig. 4E).

\section{Discussion}

In the present study, we sought to develop an effective and highly specific therapeutic approach for cervical cancer by targeting high-risk HPV E6 using CRISPR-Cas9 and the AAV vector. We previously performed screening to identify AAV vector serotypes that have the highest efficiency for gene transfer into cervical cancer cells, and demonstrated that the AAV serotype 2 vector was the most effective (14). 
A

PAM sgE6

AGGATCCAACACGGCGACCCTACAAGCTACC wt

AGGATCCAACA-------------------AGCTACC -13

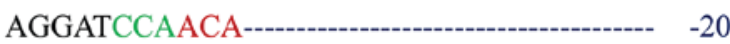

AT
AGGATCCAACACGGCGACCCTACAAGCTACC +2

CG

AGGATCCAACACGGCGACCCTACAAGCTACC +2

CGG

AGGATCCAACACGGCGACCCTACAAGCTACC +3

B

D

E
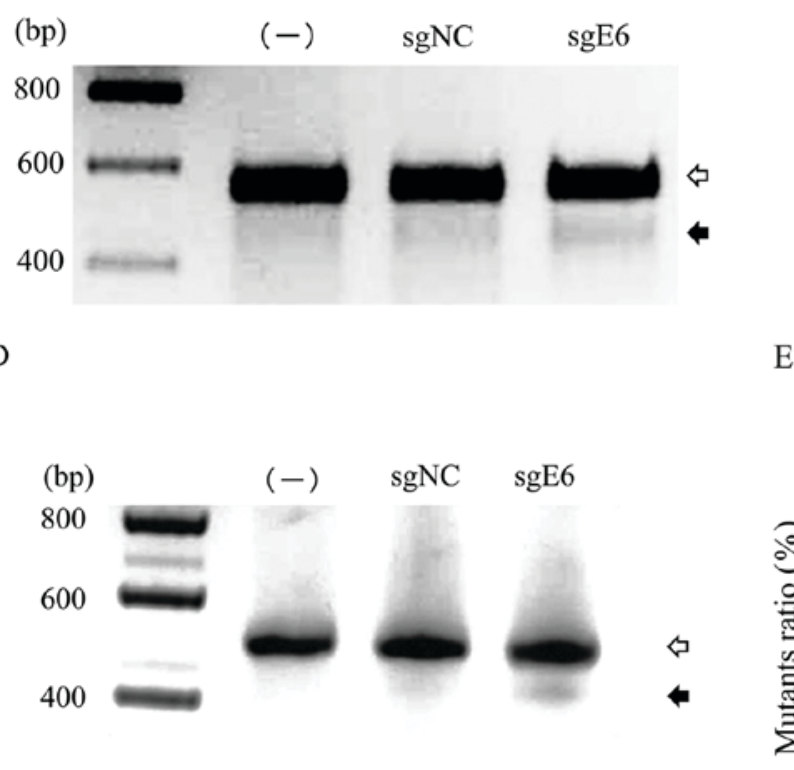

$\mathrm{C}$
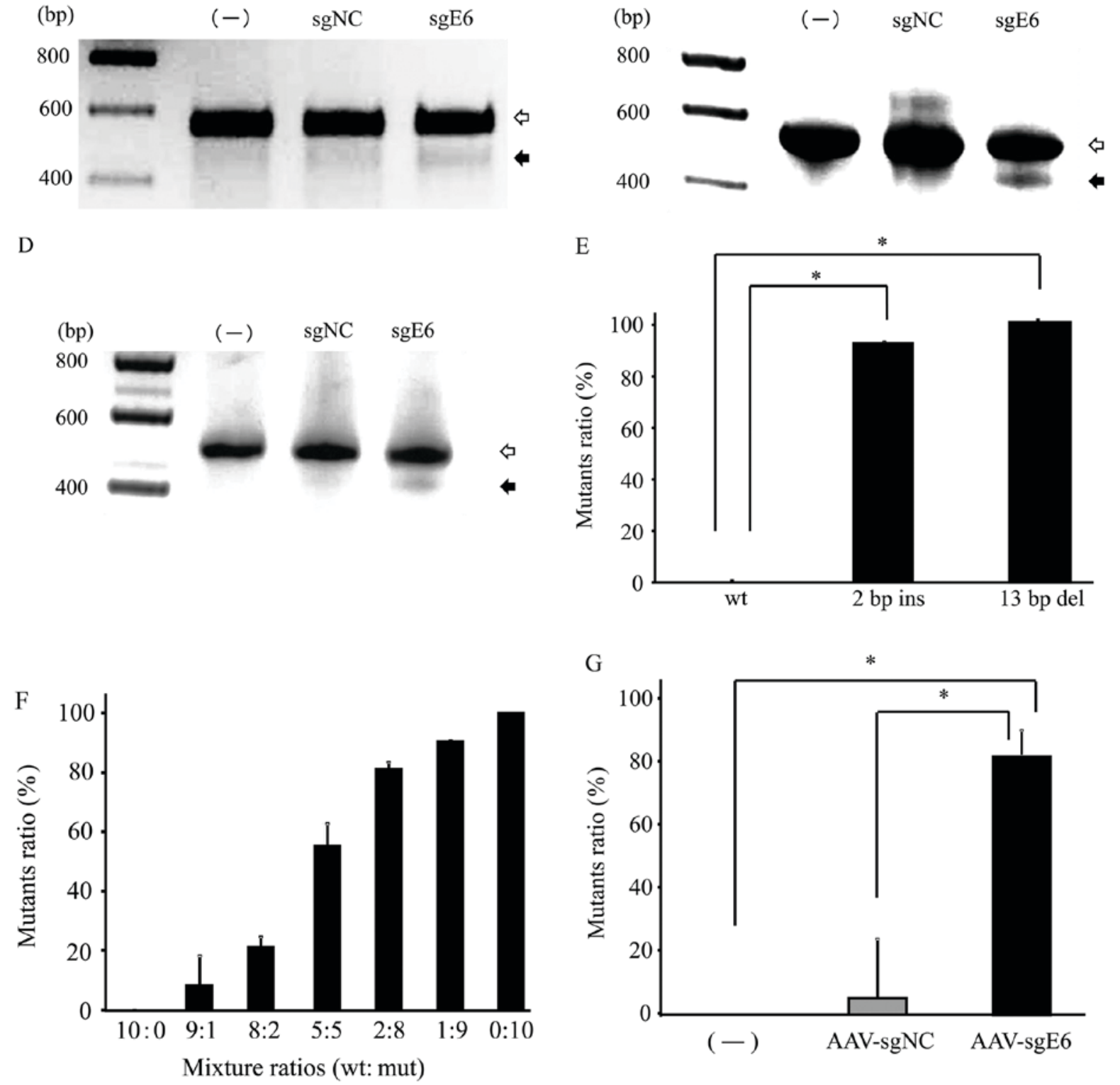

G

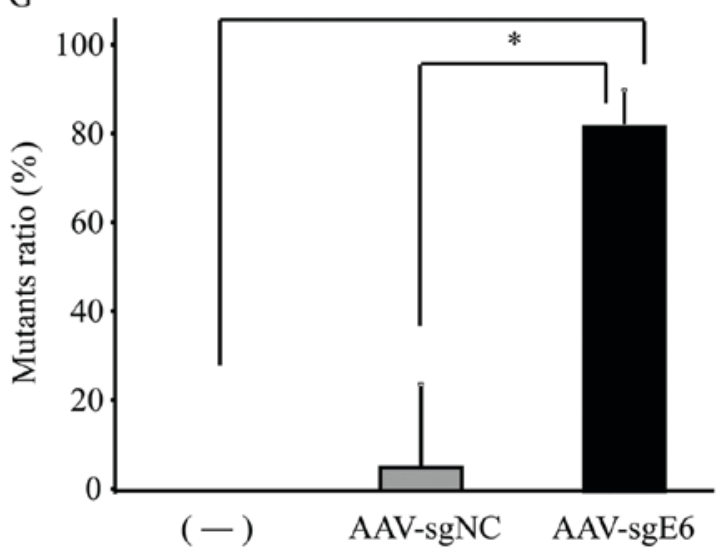

Figure 2. Detection of mutations in the E6 gene. (A) Detection of mutations in the E6 gene in Cas9-expressing HeLa cells by Sanger sequencing performed $48 \mathrm{~h}$ following AAV-sgE6 transduction. Detection of mutations in the E6 gene in Cas9-expressing cervical cancer cells by the T7 Endonuclease 1 assay performed $48 \mathrm{~h}$ following AAV-sgE6 transduction. Horizontal lines indicate base deletions. (B) HeLa/Cas9, (C) HCS-2/Cas9 and (D) SKG-I/Cas9. White arrows indicate non-cleaved products; black arrows indicate cleaved products. Validation was performed using qPCR to measure the CRISPR-Cas9-induced E6 mutation rate. The E6 mutation rate was measured in Cas9-expressing cervical cancer cells $48 \mathrm{~h}$ following AAV-sgE6 transduction. (E) Mutation rates were calculated based on the RQ. The mutation rate was calculated as: (1-RQ) x100 (\%). (F) Wild type and mutated plasmid vectors were mixed at varying ratios (10:0, 9:1, 8:2, 5:5, 2:8, 1:9 and 0:10) and used as templates. Mutation rates were calculated based on the RQ for different mixtures of plasmid vectors. DNA extracted from the Cas9-expressing cervical cancer cells $48 \mathrm{~h}$ following AAV-sgE6 transduction was used as a template for qPCR. The rate of E6 mutations in (G) HeLa/Cas9. 

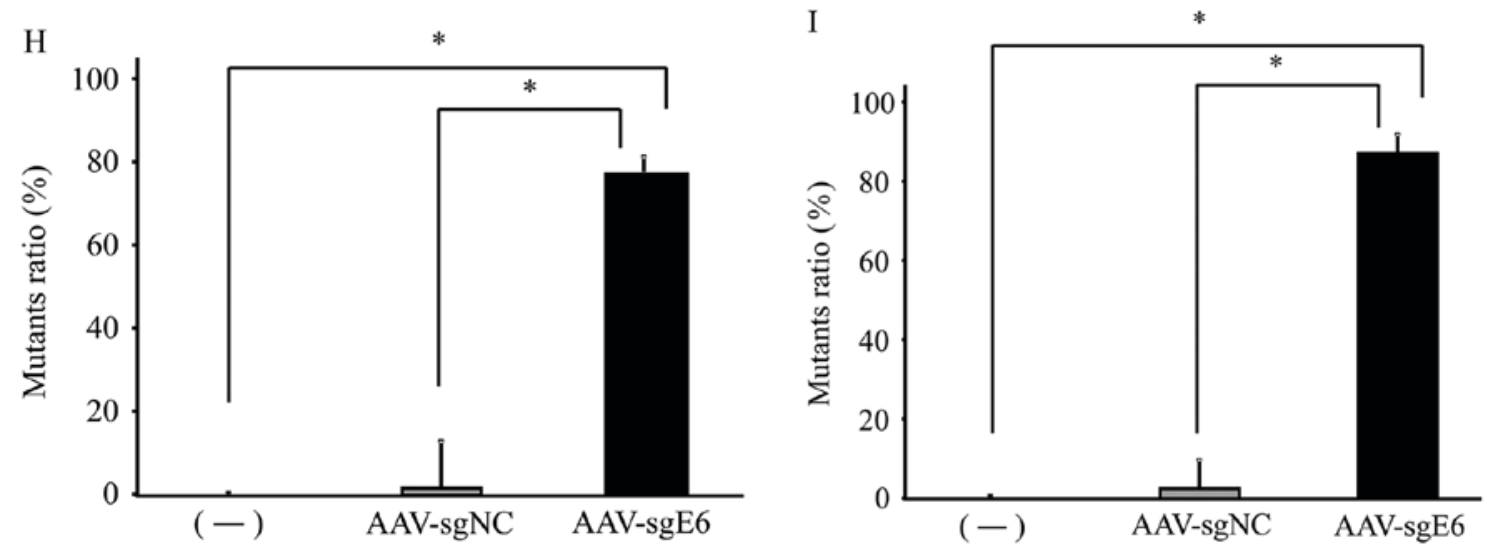

Figure 2. Continued. The rate of E6 mutations in (H) HCS-2/Cas9 and (I) SKG-I/Cas9. Results are expressed as the mean \pm standard deviation. "P $<0.05$, as indicated. PAM, protospacer-adjacent motif; sgE6, E6 target sequence; wt, wild-type; qPCR, quantitative polymerase chain reaction; 2 bp ins, E6 gene with a 2 bp insertion; 13 bp del, E6 gene with a 13 bp deletion; RQ, relative quantification; AAV, adeno-associated virus; Cas9, CRISPR associated protein 9; sg, single guide; PAM, protospacer adjacent motif; $\mathrm{NC}$, negative control.
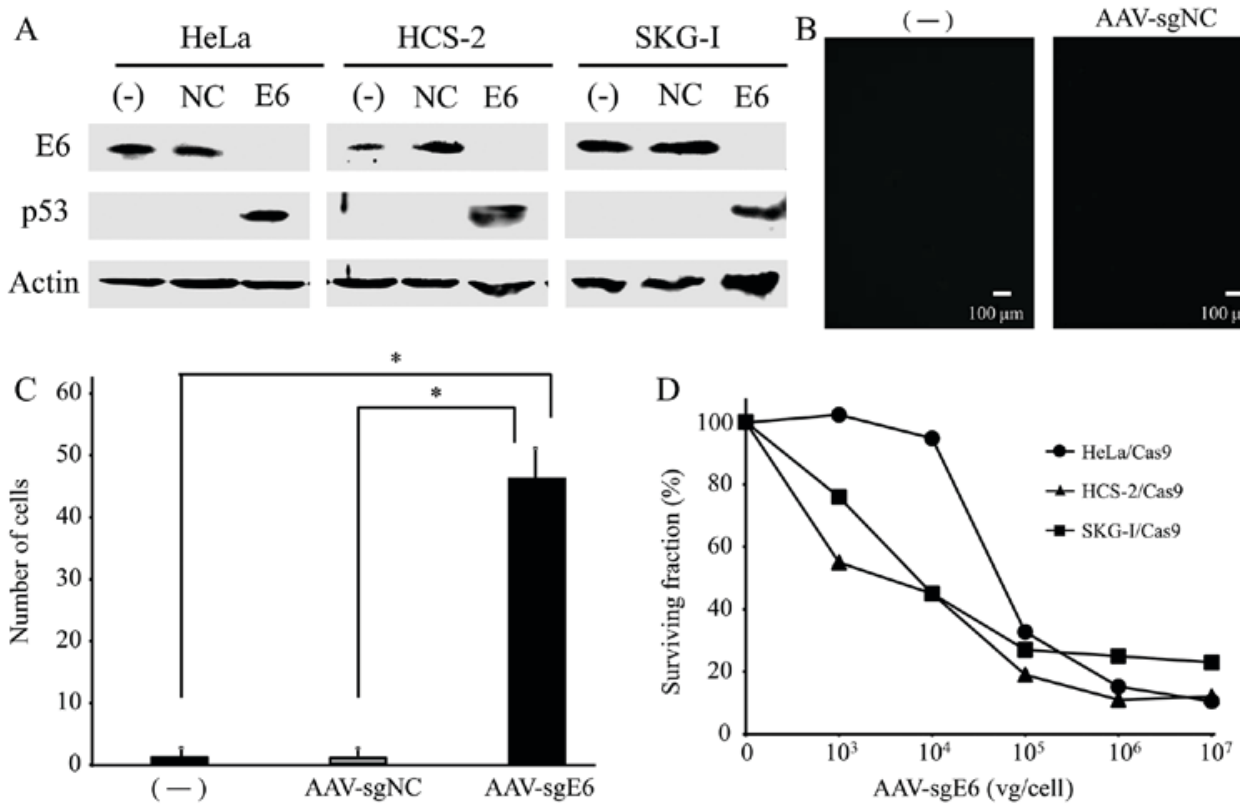

Figure 3. In vitro experiments following AAV-sgE6 transduction. (A) Expression of E6 and p53 in the three Cas9-expressing cell lines was measured by western blotting performed $48 \mathrm{~h}$ post-transduction with either AAV-sgE6 or AAV-sgNC. E6 expression was reduced, whereas p53 expression was increased in all three cell lines. Apoptosis following AAV-sgE6 transduction: (B) Apoptosis was measured in Cas9-expressing HeLa cells $48 \mathrm{~h}$ post-transduction with either AAV-sgE6 or AAV-sgNC. Scale bars, $100 \mu \mathrm{m}$. Apoptotic cells were identified by immunofluorescent staining. (C) The number of fluorescence-positive cells was counted per high-power field. " $\mathrm{P}<0.05$, as indicated. (D) In vitro cell growth following AAV-sgE6 transduction. The WST-1 assay was performed to measure the number of viable cells $72 \mathrm{~h}$ following AAV-sgE6 transduction. AAV-sgE6 suppressed tumor growth with increases in viral vector particles in all three Cas9-expressing cell lines. Results are expressed as the mean \pm standard deviation. AAV, adeno-associated virus; Cas9, CRISPR associated protein 9; sg, single guide; NC, negative control.

Based on this data, we selected AAV type 2 as the vector in the present study. We first constructed the Cas9-expressing AAV vector and observed that Cas9 was not transferred into cervical cancer cells (data not shown). The length of genomic sequence that can be packaged into the $\mathrm{AAV}$ vector is limited to approximately $5 \mathrm{~kb}(22)$. Vector production efficiency significantly decreases if longer sequences are utilized. The length of Cas9 used in this study was approximately $4.2 \mathrm{~kb}$ and exceeded $5 \mathrm{~kb}$ when the promoter and PolyA sequences were included; thus, it may not have been transferred effectively using the AAV vector. Therefore, as an alternative to using the AAV vector to introduce Cas9, we established cervical cancer cell lines that constitutively express Cas9 to perform our study. We demonstrated that the growth rate of Cas9-expressing cells was not different from that of wild type cells, confirming that Cas9 expression does not impact the growth of cervical cancer cells. As Cas9 knock-in mice that express Cas9 ubiquitously did not show any phenotypic changes (23), the expression of Cas9 might not have any influence on mammalian cells.

We then constructed the sgRNA-expressing AAV vector to target E6. We used an online service to select the target E6 sequence, and constructed the AAV vector (AAV-sgE6) that expresses the sgRNA containing the target sequence driven by the U6 promoter. Transduction of AAV-sgE6 into 

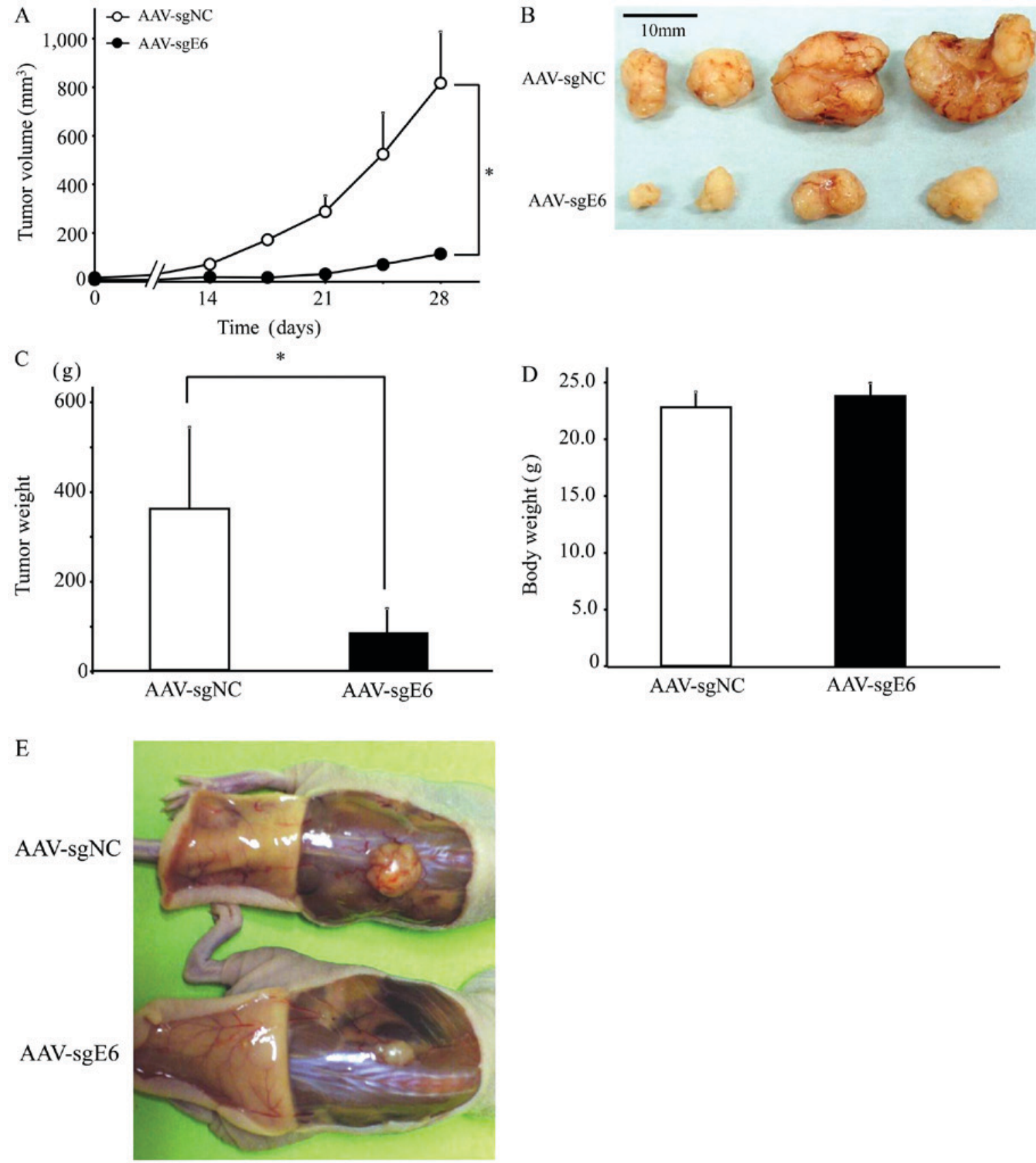

Figure 4. In vivo experiments following AAV-sgE6 transduction. (A) Growth of Cas9-expressing SKG-I tumors inoculated subcutaneously into mice. The AAV vector was injected immediately following tumor injection. (B) SKG-I tumors 28 days following the injection of AAV vectors. Scale bar, $10 \mathrm{~mm}$. (C) Weights of SKG-I tumors 28 days following the injection of AAV vectors. (D) Body weights of mice 28 days following the injection of AAV-sgE6 or AAV-sgNC. (E) Gross observations of the tumor area 28 days following the injection of AAV-sgE6 or AAV-sgNC. Results are expressed as the mean \pm standard deviation. ${ }^{*} \mathrm{P}<0.05$, as indicated. AAV, adeno-associated virus; sg, single guide; $\mathrm{NC}$, negative control.

Cas9-expressing cervical cancer cells resulted in multiple mutations in E6 as detected by Sanger sequencing. This suggested that E6-targeting by CRISPR-Cas9 may induce mutations in the E6 gene in cervical cancer cells.

The T7E1 assay is the most commonly used method for the detection of mutations induced by CRISPR-Cas9. Using this assay, we observed a new band indicating the presence of mutations in AAV-sgE6-infected cells. That said, the T7E1 assay is complex to set up as it involves multiple steps, and is not quantitative. Thus, qPCR was used to detect the mutation rate (20) by mismatch PCR. A primer pair (Mut primers) of which a part of the $3^{\prime}$ end of one primer corresponds to a part of the target
E6 sequence was constructed. In addition, another primer pair (CTL primers) was constructed such that it corresponded to another sequence distant from the target E6 sequence. When the Mut primers are used for qPCR, amplification efficiency is significantly reduced due to mismatches caused by the mutations in the sequence induced by CRISPR-Cas9. On the other hand, amplification efficiency is not affected by the presence of mutations when CTL primers are used. Thus, by comparing the RQ value of qPCR using Mut and CTL primers, it is possible to quantify the mutation rate as well as detect mutations in the target sequence. We used the plasmid vector carrying the E6 sequence with mutations identified by Sanger 
sequencing to validate this method. Our data demonstrated a significant reduction in RQ in the Mut primer group when qPCR was performed using the mutated plasmid vector as a template. Furthermore, we performed qPCR using a mixture of plasmid vectors as a template to examine whether the assay is quantitative. The template was produced by mixing vectors that carry non-mutated and mutated E6 sequences at different ratios. Our data revealed that the rate of $R Q$ reduction in the Mut primer group relative to the CTL group was equivalent to the proportion of the mutated vectors in the plasmid mixture. This finding validated the qPCR method as a quantitative method to detect CRISPR-Cas9-induced mutations in E6 and to quantify the rate of mutations. We used this method to detect mutations in E6 and determined the mutation rate in Cas9-expressing cells transduced with AAV-sgE6. E6 mutations were identified in all cell lines, and the frequency of these mutations was significantly higher than that expected with the T7E1 assay.

Next, we examined the expression of E6 and related factors using western blotting, and found decreased expression of E6, and increased expression of p53 in all three cervical cancer cell lines with AAV-sgE6. Furthermore, using Annexin V as a target, we observed that apoptosis was induced in these cells. In high-risk HPV-positive cervical cancer, p53 has no mutations $(24,25)$, and is normally degraded by E6 $(6,7)$ resulting in the inhibition of apoptosis. Our data suggest that the suppression of E6 expression by Cas9 and AAV-sgE6 led to the alteration of this pathway, resulting in the induction of apoptosis.

We subsequently examined the effects of E6-targeting CRISPR-Cas9 on the growth of cervical cancer cells in vitro, and demonstrated that AAV-sgE6 suppressed tumor cell growth in a dose-dependent manner. Based on this in vitro observation, we examined the effects of E6-targeting CRISPR-Cas9 in an in vivo model of cervical cancer, and found that tumor growth was suppressed significantly in mice injected with AAV-sgE6 as compared with those with AAV-sgNC. Using the qPCR method described above, we found no mutations in E6 in DNA extracted from tumors in the AAV-sgE6 group. This suggests that the remaining tumors may not have been transduced by the vector, or may have been formed by tumor cells that did not undergo genome editing following gene delivery. Thus, in order to enhance the efficacy of this approach, it may be necessary to increase the amount of vectors or its frequency of injection, and/or package multiple sgRNAs targeting several E6 sequences. A recent study revealed the presence of KIAA0319L, a receptor essential for AAV transduction of human cells (26). Use of this receptor may improve the transduction efficiency of AAV vectors.

Previous in vitro and ex vivo studies reported that E6 expression can be knocked out effectively in cervical cancer by genome editing techniques (27-30). In addition, in vivo experiments, the effect that E6 knockout enhances sensitivity to an anticancer drug has been reported (31). In the present study, we further demonstrated its high tumor-suppressing effects in vivo using a combination of CRISPR-Cas9, a relatively simple and effective genome editing technique, and the AAV type 2 vector, which has high transduction efficiency for cervical cancer cells.

With respect to side effects, weight loss was not observed in mice injected with AAV-sgE6 and there were no abnormal findings in the tissue around the injection site. The lack of apparent side effects may be because 1) AAV vectors are not pathogenic, and 2) E6-targeting CRISPR-Cas9 did not affect the normal tissue as E6 is only expressed in cervical cancer cells. Thus, our findings indicate that E6-targeting CRISPR-Cas9 is a safe therapeutic approach that does not affect normal tissue.

In order to translate the E6-targeting CRISPR-Cas9 approach into clinical practice, its effects may be tested initially in patients with precancerous lesions, such as CIN 2/3, or in those with early stage invasive cancers. Currently, surgical approaches, including cervical conization and total hysterectomy, are the only options to treat patients in these early stages of cancer development $(32,33)$. Cervical conization is often selected for younger patients to preserve their fertility (34). However, compared with healthy individuals, the risk of cervical cancer is still higher by 4-times after cervical conization (35). Thus, there are concerns associated with the low curability of cervical conization. Furthermore, patients who undergo cervical conization often develop reproductive or perinatal complications, such as miscarriage and premature birth, due to the removal of a portion of the endocervical canal and consequent infections in the uterine cavity (34). Thus, there is a need to develop an alternative, effective approach that is less invasive. As these precancerous and early stage lesions are localized, our approach may be the most effective strategy. Moreover, as young individuals and those who desire to have children are most affected by cervical cancer, treatment must be safe for these individuals. Although we did not observe any apparent side effects, further investigation is needed to ensure that the CRISPR-Cas9 approach can be safely performed for future clinical translation.

The effects of the CRISPR-Cas9 approach are localized, and therefore unlikely to be curative for advanced cervical cancer patients with widespread invasion and metastasis. However, it may be effective in shrinking bulky tumors, and thus may be used as an alternative to neoadjuvant chemotherapy to facilitate surgery and to increase the efficacy of radiation therapy. Furthermore, this approach may be applied to some recurrent tumors that are localized.

We demonstrated the successful transfer of E6-targeting sgRNA into cervical cancer cells by the AAV vector. Cas9 derived from Streptococcus pyogenes (SpCas9) was used in this study because it has been used the most for genome editing since its first report in 2013. As the protospacer adjacent motif (PAM) sequences for SpCas9 (NGG) that determine the target sequence are relatively short, it allows for selection of many target sequences. However, as described above, packaging of SpCas9 into the AAV vector is challenging due to its low packaging capacity. A recent study reported that Cas9 from Staphylococcus aureus (SaCas9) can be packaged effectively into the AAV vector due to its shorter length $(3.3 \mathrm{~kb})(36)$. This suggests that it is possible to package both SaCas9 and sgRNA into the AAV vector, which, in theory, should enable genome editing and E6-targeted therapy for cervical cancer. We are currently constructing an AAV vector containing SaCas9 and E6-targeting sgRNA to test its effects on cervical cancer cells.

In conclusion, we report that the CRISPR-Cas9 approach targeting high-risk HPV E6 may be a highly selective and effective therapeutic strategy for cervical cancer. 


\section{Acknowledgements}

The authors would like to thank Mrs. Miyoko Mitsu, Mrs. Satomi Fujiwara, Mrs. Akemi Takada (Division of Genetic Therapeutics, Center for Molecular Medicine, School of Medicine, Jichi Medical University, Tochigi, Japan) and Mrs. Michiko Ohashi (Department of Obstetrics and Gynecology, School of Medicine, Jichi Medical University, Tochigi, Japan) for providing excellent technical assistance.

\section{Funding}

The present study was partly supported by the JMU Graduate Student Research Award.

\section{Availability of data and materials}

The datasets used and/or analyzed during the present study are available from the corresponding author on reasonable request.

\section{Authors' contributions}

TY performed experiments, analyzed the data and wrote the manuscript. YS conceived and designed the study, performed experiments and the analyzed data. MU and RU analyzed the experimental data and provided guidance during the present study. SM and HF contributed to the interpretation of the data and revised the manuscript. HM conceived and designed the study, and critically revised the manuscript for important intellectual content. All authors read and approved the final manuscript.

\section{Ethics approval and consent to participate}

The experimental protocol was approved by the Jichi Medical University Ethics Committee (Tochigi, Japan), and strictly followed the National and Institutional Guidelines for animal experiments.

\section{Patient consent for publication}

Not applicable.

\section{Competing interests}

The authors declare that they have no competing interests.

\section{References}

1. Ferlay J, Soerjomataram I, Dikshit R, Eser S, Mathers C, Rebelo M, Parkin DM, Forman D and Bray F: Cancer incidence and mortality worldwide: Sources, methods and major patterns in GLOBOCAN 2012. Int J Cancer 136: E359-E386, 2015.

2. Siegel RL, Miller KD and Jemal A: Cancer statistics, 2016. CA Cancer J Clin 66: 7-30, 2016.

3. Woodman CB, Collins SI and Young LS: The natural history of cervical HPV infection: Unresolved issues. Nat Rev Cance 7: 11-22, 2007.

4. Boshart M, Gissmann L, Ikenberg H, Kleinheinz A, Scheurlen W and zur Hausen H: A new type of papillomavirus DNA, its presence in genital cancer biopsies and in cell lines derived from cervical cancer. EMBO J 3: 1151-1157, 1984.

5. Durst M, Gissmann L, Ikenberg H and zur Hausen H: A papillomavirus DNA from a cervical carcinoma and its prevalence in cancer biopsy samples from different geographic regions. Proc Natl Acad Sci USA 80: 3812-3815, 1983.
6. Narisawa-Saito M and Kiyono T: Basic mechanisms of high-risk human papillomavirus-induced carcinogenesis: Roles of E6 and E7 proteins. Cancer Sci 98: 1505-1511, 2007.

7. Moody CA and Laimins LA: Human papillomavirus oncoproteins: Pathways to transformation. Nat Rev Cancer 10: 550-560, 2010.

8. Da Silva DM, Eiben GL, Fausch SC, Wakabayashi MT, Rudolf MP, Velders MP and Kast WM: Cervical cancer vaccines: Emerging concepts and developments. J Cell Physiol 186: 169-182, 2001.

9. Jinek M, Chylinski K, Fonfara I, Hauer M, Doudna JA and Charpentier E: A programmable dual-RNA-guided DNA endonuclease in adaptive bacterial immunity. Science 337: 816-821, 2012.

10. Hsu PD, Lander ES and Zhang F: Development and applications of CRISPR-Cas9 for genome engineering. Cell 157: 1262-1278, 2014.

11. Mueller C and Flotte TR: Clinical gene therapy using recombinant adeno-associated virus vectors. Gene Ther 15: 858-863, 2008.

12. Grieger JC and Samulski RJ: Adeno-associated virus vectorology, manufacturing, and clinical applications. Methods Enzymol 507: 229-254, 2012.

13. Wu Z, Asokan A and Samulski RJ: Adeno-associated virus serotypes: Vector toolkit for human gene therapy. Mol Ther 14: 316-327, 2006.

14. Sato N, Saga Y, Uchibori R, Tsukahara T, Urabe M, Kume A, Fujiwara H, Suzuki M, Ozawa K and Mizukami H: Eradication of cervical cancer in vivo by an AAV vector that encodes shRNA targeting human papillomavirus type 16 E6/E7. Int J Oncol 52: 687-696, 2018.

15. Urabe M, Hasumi Y, Ogasawara Y, Matsushita T, Kamoshita N, Nomoto A, Colosi P, Kurtzman GJ, Tobita K and Ozawa K: A novel dicistronic AAV vector using a short IRES segment derived from hepatitis C virus genome. Gene 200: 157-162, 1997.

16. Matsushita T, Elliger S, Elliger C, Podsakoff G, Villarreal L, Kurtzman GJ, Iwaki Y and Colosi P: Adeno-associated virus vectors can be efficiently produced without helper virus. Gene Ther 5: 938-945, 1998.

17. Mochizuki S, Mizukami H, Kume A, Muramatsu S, Takeuchi K, Matsushita T, Okada T, Kobayashi E, Hoshika A and Ozawa K: Adenoassociated virus (AAV) vector-mediated liver- and muscle-directed transgene expression using various kinds of promoters and serotypes. Gene Ther Mol Biol 8: 9-18, 2004.

18. Xiao W, Chirmule N, Berta SC, McCullough B, Gao G and Wilson JM: Gene therapy vectors based on adeno-associated virus type 1. J Virol 73: 3994-4003, 1999.

19. Ishiwata A, Mimuro J, Mizukami H, Kashiwakura Y, Takano K, Ohmori T, Madoiwa S, Ozawa K and Sakata Y: Liver-restricted expression of the canine factor VIII gene facilitates prevention of inhibitor formation in factor VIII-deficient mice. J Gene Med 11: 1020-1029, 2009.

20. Yu C, Zhang Y, Yao S and Wei Y: A PCR based protocol for detecting indel mutations induced by TALENs and CRISPR/Cas9 in zebrafish. PLoS One 9: e98282, 2014.

21. Livak KJ and Schmittgen TD: Analysis of relative gene expression data using real-time quantitative PCR and the 2(-Delta Delta C(T)) method. Methods 25: 402-408, 2001.

22. Dong JY, Fan PD and Frizzell RA: Quantitative analysis of the packaging capacity of recombinant adeno-associated virus. Hum Gene Ther 7: 2101-2112, 1996.

23. Platt RJ, Chen S, Zhou Y, Yim MJ, Swiech L, Kempton HR, Dahlman JE, Parnas O, Eisenhaure TM, Jovanovic M, et al: CRISPR-Cas9 knockin mice for genome editing and cancer modeling. Cell 159: 440-455, 2014.

24. Crook T, Wrede D, Tidy JA, Mason WP, Evans DJ and Vousden KH: Clonal p53 mutation in primary cervical cancer: Association with human-papillomavirus-negative tumours. Lancet 339: 1070-1073, 1992.

25. Paquette RL, Lee YY, Wilczynski SP, Karmakar A, Kizaki M, Miller CW and Koeffler HP: Mutations of p53 and human papillomavirus infection in cervical carcinoma. Cancer 72: 1272-1280, 1993.

26. Pillay S, Meyer NL, Puschnik AS, Davulcu O, Diep J, Ishikawa Y, Jae LT, Wosen JE, Nagamine CM, Chapman MS and Carette JE: An essential receptor for adeno-associated virus infection. Nature 530: 108-112, 2016.

27. Hu Z, Yu L, Zhu D, Ding W, Wang X, Zhang C, Wang L, Jiang X, Shen H, He D, et al: Disruption of HPV16-E7 by CRISPR/Cas system induces apoptosis and growth inhibition in HPV16 positive human cervical cancer cells. Biomed Res Int 2014: 612823, 2014. 
28. Yu L, Wang X, Zhu D, Ding W, Wang L, Zhang C, Jiang X, Shen H, Liao S, Ma D, et al: Disruption of human papillomavirus 16 E6 gene by clustered regularly interspaced short palindromic repeat/Cas system in human cervical cancer cells. Onco Targets Ther 8: 37-44, 2014

29. Zhen S, Hua L, Takahashi Y, Narita S, Liu YH and Li Y: In vitro and in vivo growth suppression of human papillomavirus 16-positive cervical cancer cells by CRISPR/Cas9. Biochem Biophys Res Commun 450: 1422-1426, 2014.

30. Kennedy EM, Kornepati AV, Goldstein M, Bogerd HP, Poling BC, Whisnant AW, Kastan MB and Cullen BR: Inactivation of the human papillomavirus E6 or E7 gene in cervical carcinoma cells by using a bacterial CRISPR/Cas RNA-guided endonuclease. J Virol 88: 11965-11972, 2014.

31. Zhen S, Lu JJ, Wang LJ, Sun XM, Zhang JQ, Li X, Luo WJ and Zhao L: In vitro and in vivo synergistic therapeutic effect of cisplatin with human papillomavirus16 E6/E7 CRISPR/Cas9 on cervical cancer cell line. Transl Oncol 9: 498-504, 2016

32. Sevin BU, Nadji M, Averette HE, Hilsenbeck S, Smith D and Lampe B: Microinvasive carcinoma of the cervix. Cancer 70 : 2121-2128, 1992
33. Massad LS, Einstein MH, Huh WK, Katki HA, Kinney WK, Schiffman M, Solomon D, Wentzensen N and Lawson HW; 2012 ASCCP Consensus Guidelines Conference: 2012 updated consensus guidelines for the management of abnormal cervical cancer screening tests and cancer precursors. Obstet Gynecol 121: 829-846, 2013.

34. Bevis KS and Biggio JR: Cervical conization and the risk of preterm delivery. Am J Obstet Gynecol 205: 19-27, 2011.

35. Melnikow J, McGahan C, Sawaya GF, Ehlen T and Coldman A: Cervical intraepithelial neoplasia outcomes after treatment: Long-term follow-up from the British Columbia Cohort Study. J Natl Cancer Inst 101: 721-728, 2009.

36. Ran FA, Cong L, Yan WX, Scott DA, Gootenberg JS, Kriz AJ, Zetsche B, Shalem O, Wu X, Makarova KS, et al: In vivo genome editing using Staphylococcus aureus Cas9. Nature 520: 186-191, 2015.

(7) $\Theta$ This work is licensed under a Creative Commons Attribution-NonCommercial-NoDerivatives 4.0 International (CC BY-NC-ND 4.0) License. 